\title{
Neurocysticercosis
}

\section{Five new things}

\author{
Arturo Carpio, MD* \\ Agnès Fleury, MD, PhD* \\ W. Allen Hauser, MD*
}

\section{Summary}

Neurocysticercosis is the most common parasitic brain disease worldwide. Its clinical heterogeneity is related to localization, number and stage of evolution of the parasites, sex, age, and intensity of the host brain inflammatory reaction. In addition to the localization of the parasite, inflammation is the main phenomenon responsible of symptomatology. Acute symptomatic seizures are the most common symptom in patients with parenchymal parasites, but most do not evolve into epilepsy. Neurocysticercosis diagnosis is based mainly on neuroimaging. New imaging techniques have improved detection of the scolex and visualization of cysts in the extraparenchymal spaces. Immunologic testing can be useful, particularly when imaging is equivocal. Based on disappearance of parasites, antihelminthic drugs as currently used are effective in approximately one-third of patients with parenchymal viable cysts.

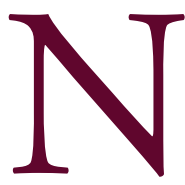

eurocysticercosis (NCC), caused by the

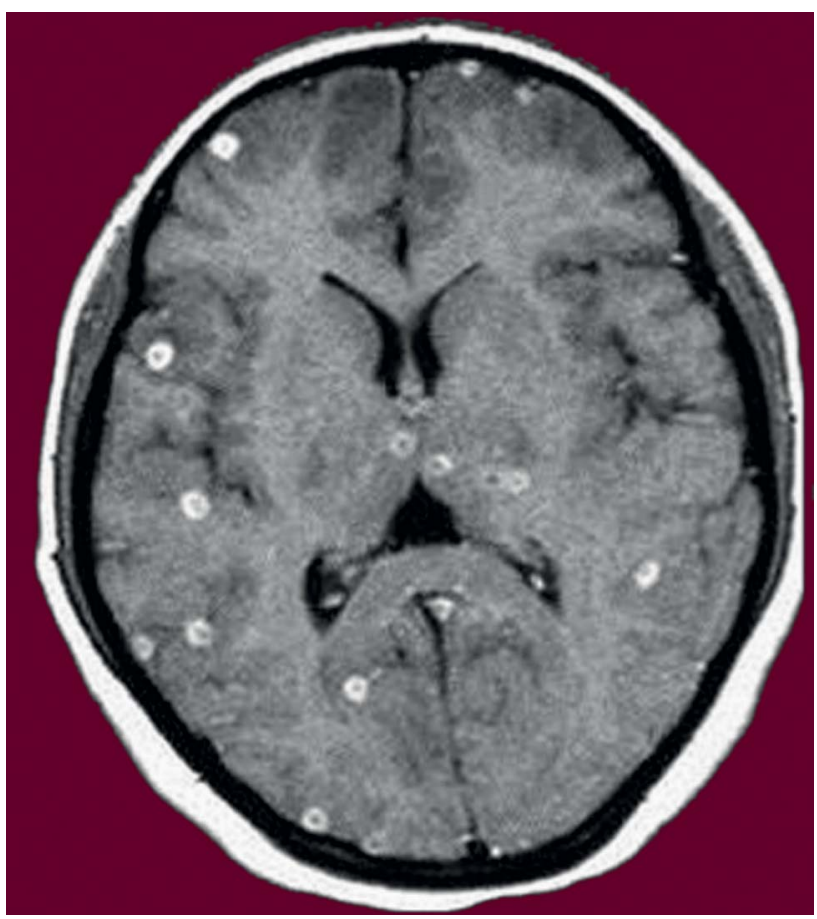
encysted larva of the tapeworm Taenia solium, is still a health problem in the current century. It is endemic in countries with poor sanitation, and is increasingly being reported in developed countries due to globalization and immigration. ${ }^{1}$ Prognosis of NCC has changed in the past 50 years, principally due to the improvement of imaging techniques and to the use of antihelminthic drugs, but this disease remains the cause of unacceptable morbidity and mortality. In this article, we briefly present 5 aspects of the disease, highlighting the progress made but also stressing persisting problems: heterogeneity of clinical manifestation, inflammation and NCC, epilepsy and NCC, diagnosis, and treatment.

\section{Heterogeneity of neurocysticercosis}

A major characteristic of NCC is heterogeneity, which is seen at a number of levels. ${ }^{2}$ Clinically, CNS parasites can be asymptomatic (apparently the case in most of those affected), or can

*These authors contributed equally to this work.

Research Department (AC), University of Cuenca, Cuenca, Ecuador; G.H. Sergievsky Center (AC, WAH), Faculty of Medicine, Columbia University, New York, NY; and Instituto de Investigaciones Biomédicas (AF), UNAM Instituto Nacional de Neurologia y Neurocirugía, SS, Mexico City, Mexico.

Correspondence to: arturocarpio@etapanet.net 
produce a great variety of symptoms, including headaches, dizziness, seizures, focal neurologic deficit, psychiatric symptoms, or severe intracranial hypertension. This clinical heterogeneity is due to variation in the localization, number, and stage of evolution of the parasites as well as to the intensity of the inflammatory reaction.

Another level of heterogeneity is the response to antihelminthic drugs (AHD), albendazole or praziquantel. Some parasites will be eradicated while others will resist the treatment.

Both host and parasite factors seem to be involved in this heterogeneity. The intensity of the immune reaction is greater in women compared to men. There is a decrease in host inflammatory response across age groups, with a higher proportion of calcified and inflammatory parasites being identified in children. ${ }^{2}$ Host genes are also involved in the heterogeneity. In endemic areas, aggregation of cases has been found in families and polymorphisms have been found to be associated with symptoms in patients with calcified NCC (MMP-9) and parenchymal NCC (TLR-4). ${ }^{3,4}$ Genetic differences also exist between parasites, although for the moment, evidence of their relevance in the heterogeneity are lacking — at least in pigs. ${ }^{5}$ The genome of $T$ solium will soon be sequenced, and this may improve our knowledge of mechanisms of pathogenesis and the genetic role in disease heterogeneity. ${ }^{6}$ Clinical and radiologic presentation varies between endemic countries: in India, single enhanced granuloma are more frequent than in Latin America; in India and Africa, subarachnoid and ventricular localizations are less frequently identified than in Latin America, while subcutaneous and muscular localizations are more common. These differences point to the relevance of the genetic status of the host or parasite. ${ }^{2}$ Multidisciplinary investigations in this area may provide clues to factors involved in clinical severity, leading to improvement in prevention, treatment, and patient management.

\section{Inflammation and neurocysticercosis}

The inflammatory reaction associated with NCC is complex and not completely understood. Inflammation is necessary for lesion resolution but is also causal for pathology associated with both short- and long-term complications. In brain parenchyma, inflammation around the parasites is the main phenomenon triggering seizures. This occurs frequently in degenerating cysts and calcified lesions. ${ }^{3}$ For extraparenchymal parasites, inflammation of the CSF space is frequently intense, causing arachnoiditis, ependymitis, vasculitis, and cranial nerve entrapment, and commonly requires a ventriculoperitoneal shunt. ${ }^{7}$

Immunohistochemical studies of the inflammatory infiltrate around the cysticerci have revealed specific immunoglobulin M and plasmatic cells, NK lymphocytes, macrophages, granulocytes, and T cells, which vary based upon parasite stage and location. In CSF, there is evidence of inflammation based on pleocytosis (mainly lymphocytes) with increased levels of all immunoglobulin G subclasses, interleukin-6, interleukin-5, interleukin-10, protein, and eosinophils. In blood, decreased proliferation of specific cells and increase of the proportion of $T$ regulatory cells has been observed. ${ }^{8}$

Corticosteroids are frequently used to modify the inflammatory response. The immune-modulatory action of corticosteroids is the result of inhibitory effects on immune cells, inhibition of lymphocyte binding to endothelial cells, increased apoptosis of $\mathrm{T}$ and $\mathrm{B}$ cells, inhibition of Tolllike receptor pathway in antigen-presenting cells, and downregulation of dendritic cell migration and maturation. ${ }^{9}$ Although corticosteroids frequently improve symptoms, the high doses and long duration of administration is a source of undesirable side effects. In some patients, in whom steroids cannot be successfully tapered, methotrexate and azathioprine have been used. Of course, these treatments also lead to numerous side effects. Dextrochlorpheniramine has also been used but its effectiveness has not been confirmed. ${ }^{10}$

Inflammation is a necessary part of the death process of the parasite. Inflammation is minimal around vesicular (viable) parasites, while an inflammatory reaction, varying in intensity between patients, is always present in degenerating cysts. In TLR2-/- KO mice and a murine model of NCC (Mesocestoides corti), a decrease of inflammatory reaction was associated with a higher 


\section{In spite of the clinical heterogeneity and} variability in the clinical forms of NCC, all studies agree that seizures are the most

\section{common symptom, occurring in 60\%-90\% of patients.}

susceptibility to the parasites and to an increase in the number of parenchymal cysts. ${ }^{11}$ The control of the inflammatory reaction, necessary to avoid severe complications, may also promote the survival of parasites. Patients not responding to AHD treatment or with emergence of new parasites after treatment are presumably patients with high-dose long-term corticosteroids treatment. ${ }^{7}$ Corticosteroids may not be the best product to use to modify inflammation. Future investigations must be oriented 1) to identify specific inflammatory mediators involved in the inflammatory-mediated pathology and 2) to identify targeted anti-inflammatory therapy, without undesirable side effects.

\section{Neurocysticercosis and epilepsy}

In spite of the clinical heterogeneity and variability in the clinical forms of NCC, all studies agree that seizures are the most common symptom, occurring in $60 \%-90 \%$ of patients. ${ }^{1}$ Recent studies have confirmed that children are more likely to have NCC-related seizures than adults. ${ }^{12}$ Seizures may occur at any evolutionary stage of the parasite, but seizures in the context of edema and a degenerative lesion should be considered acute symptomatic, even if they occur many months after presentation. ${ }^{13}$ It is appropriate to continue antiepileptic drugs (AED) until resolution of the acute lesion, at which time antiseizure medication may be discontinued. For the majority of people with acute symptomatic seizures, epilepsy does not follow. ${ }^{1}$

Recurrent seizures occurring after resolution of edema or calcification of the degenerating cyst should be considered unprovoked (epilepsy). In these cases, long-term antiseizure medication is warranted. Many studies have reported an association between epilepsy and NCC based on positive serum antibodies to $T$ solium/cysticercosis ${ }^{1}$; however, the presence of antibodies indicates previous exposure to or infection with the parasite, but not necessarily brain infection. Cysticercosis and epilepsy are both common diseases in many endemic countries. Because of the high prevalence of each condition, coexistence of NCC antibodies does not necessarily imply causation of epilepsy.

Parasite location is frequently remote from the apparent epileptogenic region. There is no correlation between the NCC burden of lesions and the severity of the epilepsy. The mechanism by which the calcified neurocysticercal lesions (CNL) cause seizures or epilepsy is related to residual perilesional gliosis that results in chronic epileptogenic foci ${ }^{3}$; however, CNL are frequently encountered on CT scans of asymptomatic individuals. Edema surrounding CNL after seizures has been described'; this is a very rare event in clinical practice and it has been suggested that release of cysticercal antigens from the calcified lesions lead to inflammation and perilesional edema. It is not clear whether this edema is causal or a consequence of the seizure.

NCC patients with acute symptomatic seizures have a good prognosis in terms of remission of seizures. ${ }^{1}$ The risk of seizures is substantial as long as there is persistence of edema around the degenerating lesion and this risk decreases after the cysts have resolved or become inactive. ${ }^{1}$

\section{Diagnoses of neurocysticercosis}

Clinical manifestations of NCC are determined by parasite location within the CNS, the evolutionary phase of the parasite, and the patient's immunologic response. They are not specific and diagnosis cannot be established on clinical grounds alone. In the parenchyma, seizures, 
To be useful in clinical practice, an

immunodiagnostic test should be specific for the larval phase of the parasite, specific for localization in the CNS, and should differentiate viable and nonviable forms of the parasite.

headache, focal motor deficits, and psychiatric and cognitive symptomatology are the most frequent clinical manifestations, ${ }^{1}$ while in cysts in extraparenchymal locations, intracranial hypertension is more common. ${ }^{7}$

To be useful in clinical practice, an immunodiagnostic test should be specific for the larval phase of the parasite, specific for localization in the CNS, and should differentiate viable and nonviable forms of the parasite. Because cysticerci can invade other organs besides the CNS, this ideal test is not currently available. Detection of specific antibodies in sera using ELISA and enzyme-linked immunoelectrotransfer blot (EITB) assay do not differentiate between viable and degenerated parasites, and are not specific to CNS localization. ${ }^{2}$ EITB seems to be more effective than ELISA in sera (around 90\% sensitivity and $99 \%$ specificity for cysticercosis), although its cost prevents its regular utilization in most endemic countries. ${ }^{1}$ In patients with solitary cysticercus granuloma, the positivity of EITB in sera is low, i.e., $20 \%$ $80 \% .^{7}$ Detection of specific antigens in sera by ELISA in patients with parasites located in the subarachnoid space or the ventricular system is a specific sign of parasite viability and may be used to evaluate treatment response. Specificity of these tests will increase using CSF, but lumbar puncture is not routine in endemic countries and may be dangerous in individuals at risk for increased intracranial pressure. CSF PCR for $T$ solium has a high detection rate and may become a useful clinical test. ${ }^{14}$

CT and MRI are useful in diagnosis of NCC and in the evaluation of parasite evolutionary stage, as well as number and localization of lesions. Specifically, imaging procedures allow visualization of vesicular, colloidal, granular-nodular, and calcified phases of the parasite in the CNS (figure 1). ${ }^{1}$ MRI is more sensitive than CT for the detection of the scolex and for the diagnosis of extraparenchymal NCC. The characteristics of the parenchymal cysticerci have been well-described elsewhere ${ }^{1}$ and the principal differential diagnosis is cystic tumor, for which spectroscopy can be of help. Extraparenchymal NCC is more difficult to detect by imaging because the attenuation and signal intensity of the cyst's content is similar to that of CSF and the cystic wall is usually not detected, and the cysts frequently lack a scolex and are generally not enhanced after contrast administration. ${ }^{7}$ MRI techniques such as fluid-attenuated inversion recovery (FLAIR) performed 5 minutes after continuous inhalation of $100 \%$ oxygen and fast imaging employing steady-state acquisition (FIESTA) sequences permit better detection of the parasites ${ }^{7}$ (figure 2). Definitive diagnosis of NCC thus may require the conjunction of different testing modalities that can be difficult to obtain in endemic countries. Del Brutto et $\mathrm{a}^{15}$ proposed diagnostic criteria for NCC. Although this effort was important, it has not yet been validated. Modifications may be necessary, particularly for extraparenchymal NCC and for single enhancing lesions, for which these diagnostic criteria are not useful.

\section{Treatment of neurocysticercosis}

Symptomatic treatment for both parenchymal and extraparenchymal location is based upon clinical manifestations: AED for seizures, mannitol for high intracranial pressure, analgesics 


\section{Figure $1 \quad$ MRI and CT scan of parenchymal cysticerci}

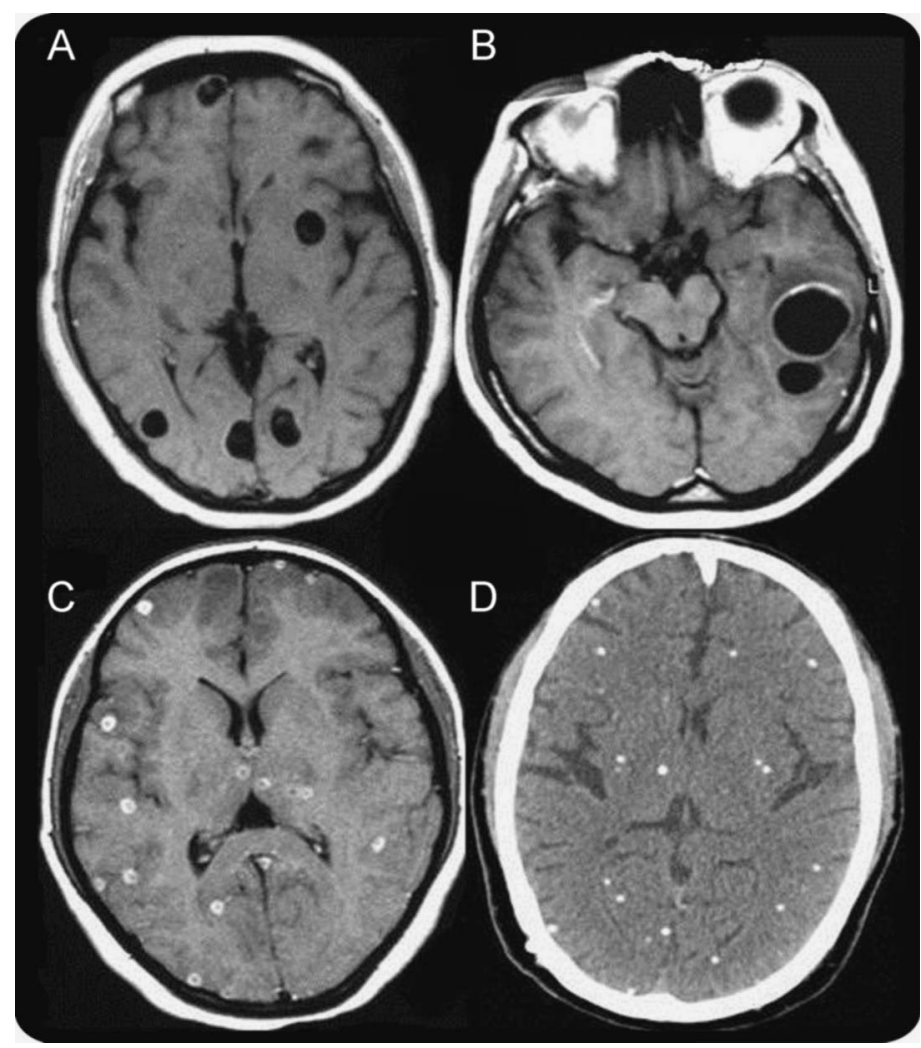

(A) MRI of vesicular cysticerci. (B) MRI of colloidal cysticerci. (C) MRI of nodular cysticerci. (D) CT scan of calcified cysticerci.

for headache. ${ }^{1}$ Steroids are often administered on the premise that they reduce inflammation and edema around dying parenchymal cysts, ${ }^{9}$ and are also recommended for treatment of large subarachnoid cysts and arachnoiditis. However, optimal dose, duration, and timing of administration of corticosteroids are unknown. ${ }^{16}$

Treatment of NCC is complex and should be individualized, based on location and viability of the parasites. The intuitive approach is to kill the parasite using available antihelminthic drugs (praziquantel, albendazole). Most publications report "reduction of the number of lesions" to measure treatment effectiveness, which is misleading. If a person with 10 viable

\section{Figure $2 \quad$ MRI of subarachnoid cysticerci}
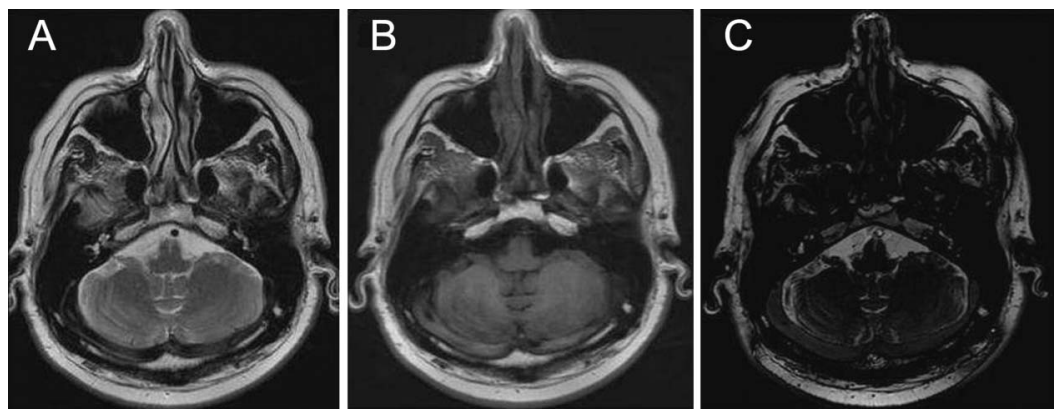

MRI: T2 (A), T1 (B), and fast imaging employing steady-state acquisition (FIESTA) (C) sequences. These images show the superiority of FIESTA sequence to visualize cysticerci in cisternal subarachnoid space. 
Figure 3 Treatment of neurocysticercosis

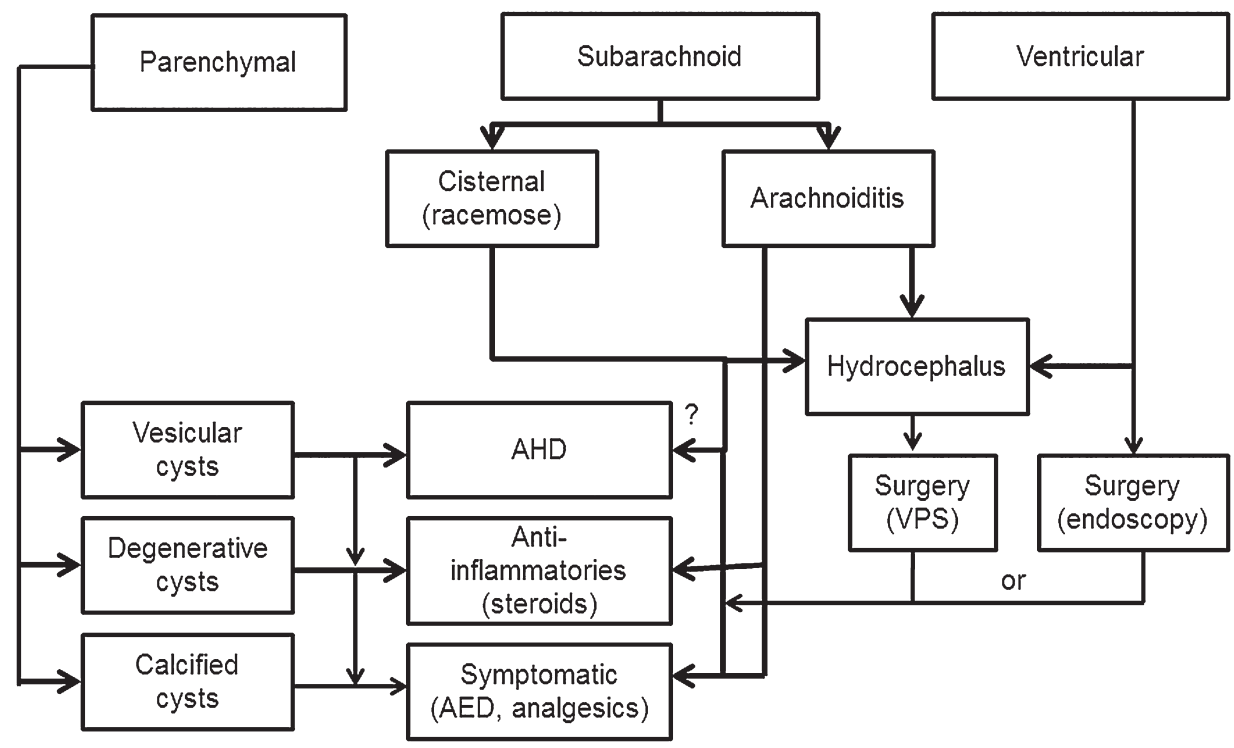

Praziquantel ( $50 \mathrm{mg} / \mathrm{kg} /$ day for 15 days), albendazole $(15 \mathrm{mg} / \mathrm{kg} /$ day for 8 days); more frequent treatment schemes used. $\mathrm{AED}=$ antiepileptic drugs; $\mathrm{AHD}=$ antihelminthic drugs; VPS = ventriculoperitoneal shunt.

cysts has been administered AHD and 8 parasites die (80\% reduction of lesion), 2 cysts still remain, which may continue to cause seizures or headache, and this intervention should not be considered successful. The appropriate endpoint to evaluate the effectiveness of AHD should be cyst disappearance. ${ }^{16}$

One meta-analysis of response to AHD in people with $\mathrm{NCC}^{17}$ concluded that studies provided evidence of a modest effect of NCC treatment, in viable parenchymal cysts, with relatively small effects of cysticidal treatment on neuroimaging endpoints (odds ratio $<2.2$ [1.10-5.01]). A Cochrane review ${ }^{18}$ concluded that in patients with viable lesions, evidence from trials of adults suggests that albendazole may reduce the number of lesions. These conclusions confirm that clinical efficacy of AHD is still in question. So far, there are no controlled clinical trials to establish definitive doses and duration of treatment.

At present, there is evidence of the real efficacy of AHD treatment for parenchymal viable cysts. In fact, according to placebo-controlled clinical trials, ${ }^{16,19}$ AHD are effective in about $30 \%$ to $40 \%$ of patients with parenchymal viable cysts, measured as disappearance of cysts in imaging studies. Some case series have reported AHD to be effective in extraparenchymal forms, whereas others report failure of treatment, even using a high dose of albendazole. ${ }^{7}$ In a double-blind placebo-controlled study, disappearance of extraparenchymal cysts with albendazole did not reach significance. ${ }^{16}$ Neurosurgical intervention should be considered for hydrocephalus requiring ventriculoperitoneal shunt or intraventricular localization of a cyst.

A common presenting form of NCC is the solitary cysticercus granuloma (SCG), which represents a single, degenerating parenchymal cysticercus cyst. Treatment approach to SCG has not been optimally defined. ${ }^{20}$ While treatment with albendazole has been shown to improve outcome in live, viable parenchymal cysticercosis, the benefits of antihelminthic treatment in patients with SCG remain uncertain, despite several randomized trials. ${ }^{20}$ Similarly, controversy exists regarding the use of corticosteroids, alone or in combination with AHD.

A recent review concerning diagnostic and therapeutic schemes for SCG concluded that the use of antihelminthic agents (specifically, albendazole in combination with corticosteroids) and corticosteroids alone have been shown to improve radiologic resolution and seizure outcome in patients with SCG, but the size of the effect was modest. ${ }^{20}$ The authors recommended large, multicenter trials with sufficiently long follow-up, comparing outcomes with the use of AHD 
with or without corticosteroids and corticosteroids alone in order to ascertain the benefits of these drugs.

In the case of SGC, the parasite is already dead and will resolve or be calcified by natural evolution of the disease. ${ }^{16}$ This lesion, regardless of its size or location, should not be biopsied or removed (with the understanding that other pathologies such as pyogenic abscess, tuberculoma, and metastatic brain tumors have been excluded). A common clinical practice is to

\section{Neurocysticercosis: Five new things}

- Host and parasite factors involved in NCC heterogeneity are beginning to be deciphered and in the future will provide new clues to better management of the disease.

- Control of the inflammatory reaction in NCC is under debate today: it can surely reduce frequency of some complications, but it can also contribute to the poor clinical evolution of some patients.

- Coexistence of cysticercus antibodies does not necessarily imply causation of epilepsy. Because of the high prevalence of each condition in endemic countries, a causal as well as fortuitous relationship between the 2 pathologies might exist.

- The use of new MRI sequences and the detection of parasite antigen have improved diagnosis of the severe extraparenchymal form of the disease.

- Current cestocidal drugs help to resolve the infection in one-third of the parenchymal viable NCC cases. This is important to pursue in the research of new therapeutic options for extraparenchymal NCC. initiate AED in patients having SCG with seizures. Available evidence suggests that withdrawal of AED after complete resolution of the SCG is safe. ${ }^{20}$

The effect of AHD on recurrence of seizures is an issue under debate. The Cochrane review found no difference when albendazole was compared to no treatment for recurrence of seizures in patients with viable parenchymal cysts ${ }^{18}$; other studies found a modest effect of albendazole in patients with degenerating cysts. ${ }^{20}$ At present, there is no good answer to the question whether a patient with a single viable cyst with no symptoms should be treated with AHD. Thus, the current information is incomplete and did not permit consensual guidelines. It is therefore recommended to conduct large, multicenter trials with sufficiently long follow-up to reach an agreement. This panorama also demonstrates the relevance for continued research to identify new and more efficient treatments for these cases.

Although simplistic, due to the heterogeneity of NCC cases and to the unanswered questions, we propose a flowchart of NCC treatment showing the management of NCC cases, according to recent studies using evidence-based recommendations $^{20}$ (figure 3).

\section{REFERENCES}

1. Carpio A. Neurocysticercosis: an update. Lancet Infect Dis 2002;2:751-762.

2. Fleury A, Escobar A, Fragoso G, Sciutto E, Larralde C. Clinical heterogeneity of human neurocysticercosis results from complex interactions among parasite, host and environmental factors. Trans R Soc Trop Med Hyg 2010;104:243-250.

3. Gupta RK, Awasthi R, Rathore RK, et al. Understanding epileptogenesis in calcified neurocysticercosis with perfusion MRI. Neurology 2012;78:618-625.

4. Verma A, Prasad KN, Gupta RK, et al. Toll-like receptor 4 polymorphism and its association with symptomatic neurocysticercosis. J Infect Dis 2010;202:1219-1225.

5. Bobes RJ, Fragoso G, Reyes-Montes MR, et al. Genetic diversity of Taenia solium cysticerci from naturally infected pigs of central Mexico. Vet Parasitol 2010;26: $130-135$.

6. Aguilar-Díaz H, Bobes RJ, Carrero JC, et al. The genome project of Taenia solium. Parasitol Int 2006; 55(suppl):S127-130.

7. Fleury A, Carrillo-Mezo R, Flisser A, Sciutto E, Corona T. Subarachnoid basal neurocysticercosis: a focus on the most severe form of the disease. Expert Rev Anti Infect Ther 2011;9:123-133.

8. Adalid-Peralta L, Fleury A, García-Ibarra TM, et al. Human neurocysticercosis: in vivo expansion of peripheral regulatory $\mathrm{T}$ cells and their recruitment in the central nervous system. J Parasitol 2012;98: 142-148.

9. Nash TE, Pretell EJ, Lescano AG, et al. Perilesional brain oedema and seizure activity in patients with calcified neurocysticercosis: a prospective cohort and nested case-control study. Lancet Neurol 2008;7: 1099-1105.

10. Agapejev S. Neurocysticercosis: the enigmatic disease. Cent Nerv Syst Agents Med Chem 2011;11: 261-284 
11. Gundra UM, Mishra BB, Wong K, Teale JM. Increased disease severity of parasite-infected TLR2-/mice is correlated with decreased central nervous system inflammation and reduced numbers of cells with alternatively activated macrophage phenotypes in a murine model of neurocysticercosis. Infect Immun 2011;79:2586-2596.

12. Kelvin EA, Carpio A, Bagiella E, et al. The association of host age and gender with inflammation around neurocysticercosis cysts. Ann Trop Med Parasitol 2009;103:487-499.

13. Beghi E, Carpio A, Forsgren L, et al. Recommendation for a definition of acute symptomatic seizure. Epilepsia 2010;51:671-675.

14. Almeida CR, Ojopi EP, Nunes CM, et al. Taenia solium DNA is present in the cerebrospinal fluid of neurocysticercosis patients and can be used for diagnosis. Eur Arch Psychiatry Clin Neurosci 2006; 256:307-310.

15. Del Brutto OH, Rajshekhar V, White AC Jr, et al. Proposed diagnostic criteria for neurocysticercosis. Neurology 2001;57:177-183.

16. Carpio A, Kelvin E, Bagiella E, et al. The effects of albendazole treatment on neurocysticercosis: a randomized controlled trial. J Neurol Neurosurg Psychiatry 2008;79:1050-1055.

17. Del Brutto OH, Roos KL, Coffey CS, Garcia HH. Meta-analysis: cysticidal drugs for neurocysticercosis: albendazole and praziquantel. Ann Intern Med 2006;45:43-51.

18. Abba K, Ramaratnam S, Ranganathan LN. Anthelmintics for people with neurocysticercosis. Cochrane Database Syst Rev 2010;17:CD000215.

19. Garcia H, Pretell E, Gilman R, et al. A trial of antiparasitic treatment to reduce the rate of seizures due to cerebral cysticercosis. N Engl J Med 2004;350:249-258.

20. Singh G, Rajshekhar V, Murthy JM, et al. A diagnostic and therapeutic scheme for a solitary cysticercus granuloma. Neurology 2010;75:2236-2245.

\section{STUDY FUNDING}

No targeted funding reported.

\section{DISCLOSURES}

The authors report no disclosures. Go to Neurology.org/cp for full disclosures.

\section{Related articles from other AAN physician and patient resources}

\section{Neurology ${ }^{\circledR} \quad \bullet \quad$ www.neurology.org}

Editorial: What causes seizures in patients with calcified neurocysticercal lesions? February 28, 2012;78:612-613.

Understanding epileptogenesis in calcified neurocysticercosis with perfusion MRI February 28, 2012;78:618-625.

Cognitive impairment and dementia in neurocysticercosis: A cross-sectional controlled study April 20, 2010;74:1288-1295.

\section{Continuum: Lifelong Learning in Neurology ${ }^{\circledR}$}

Neurocysticercosis

December 2012;1392-1416

The neurology of parasitic diseases and malaria. February 2011;113-133. 